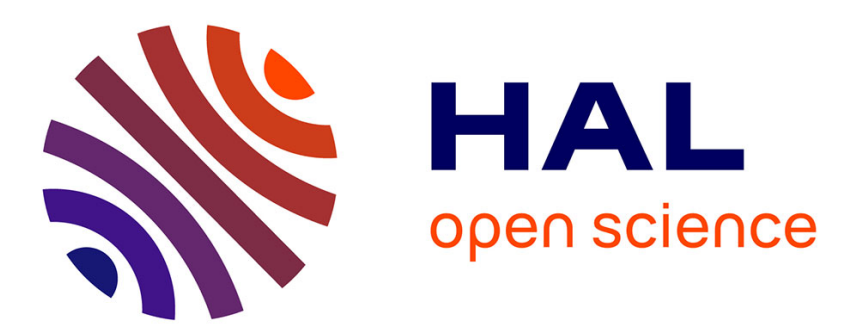

\title{
Should the beneficial impact of bezafibrate on fatty acid oxidation disorders be questioned?
}

Jean-Paul Bonnefont, Fatima Djouadi, Jean-Louis Bresson, Jean Bastin

\section{To cite this version:}

Jean-Paul Bonnefont, Fatima Djouadi, Jean-Louis Bresson, Jean Bastin. Should the beneficial impact of bezafibrate on fatty acid oxidation disorders be questioned?. Journal of Inherited Metabolic Disease, 2015, 38 (2), pp.371-372. 10.1007/s10545-014-9775-7 . inserm-02894834v2

\section{HAL Id: inserm-02894834 \\ https://www.hal.inserm.fr/inserm-02894834v2}

Submitted on 9 Jul 2020

HAL is a multi-disciplinary open access archive for the deposit and dissemination of scientific research documents, whether they are published or not. The documents may come from teaching and research institutions in France or abroad, or from public or private research centers.
L'archive ouverte pluridisciplinaire HAL, est destinée au dépôt et à la diffusion de documents scientifiques de niveau recherche, publiés ou non, émanant des établissements d'enseignement et de recherche français ou étrangers, des laboratoires publics ou privés. 


\title{
Should beneficial impact of bezafibrate on fatty acid oxidation disorders be questioned?
}

Jean Bastin, Ph.D., Jean-Paul Bonnefont, M.D., Ph.D, Fatima Djouadi, Ph.D. Jean-Louis Bresson, M.D., Ph.D.

From:

INSERM UMR-S 1124, Université Paris Descartes, Paris, France (JB and FD)

IHU IMAGINE (UMR1163), and Laboratoire hospitalier de génétique moléculaire Groupe hospitalier Necker Enfants Malades (JPB)

Université Paris Descartes, Hôpital Necker-Enfants Malades Paris, France (JLB)

\author{
Corresponding author: Jean Bastin \\ INSERM UMR-S 1124 \\ Université Paris Descartes \\ UFR Biomédicale des Saints-Pères \\ 45, rue des Saints-Pères \\ 75270 Paris cedex 06 \\ France
}

Phone: 33-1-42862219

Fax: 33-1-42863868

e-mail: jean.bastin@inserm.fr

Financial disclosure statement: the authors have no potential conflict of interest to declare. 
Inborn defects of fatty acid ß-oxidation (FAO) belong to a large group of genetic disorders affecting mitochondrial energy production, which carry significant morbidity in children and adults. Diagnosis of these disorders has greatly improved over the last decades, revealing a broad spectrum of clinical manifestations, but their pharmacological therapy is still in its infancy. As with other rare disorders, very few clinical trials have been conducted in FAO deficient patients, and maintaining research momentum is critical in developing solutions for unmet treatment needs.

Several lines of evidence suggest that bezafibrate, a widely prescribed hypolipidemic drug, could be effective for the treatment of FAO disorders. In rodents, it is well established that fibrates trigger up-regulation of mitochondrial FAO in many tissues, mediated by activation of PPAR (Peroxisome Proliferator Activated Receptors) nuclear receptors (1, 2). We, and others, have shown that exposure to bezafibrate could improve or correct mild forms of FAO deficiencies in patients' fibroblasts (3-6). Compared analysis in various genotypes revealed that a large number of missense mutations encountered in the adult forms of Carnitine palmitoyl transferase 2 (CPT2) or Very Long Chain AcylCoA Dehydrogenase (VLCAD) deficiencies were associated with beneficial responses of patient fibroblasts to bezafibrate, leading to the correction of FAO deficiency. In line with this, a 2009 open pilot clinical trial in patients with the muscular form of CPT2 deficiency demonstrated a stimulation of muscle FAO capacities after six months treatment by bezafibrate, accompanied with improvements in the muscular symptoms and clinical condition of the patients $(7,8)$.

These conclusions were recently challenged by the results of a second trial in five CPT2- and five Very Long Chain AcylCoA Dehydrogenase (VLCAD) -deficient patients. This 3-month, randomized, double-blind, crossover study, published by Orngreeen et al. in a recent issue of Neurology, came to the conclusion that bezafibrate improved neither FAO nor the disease manifestations (9). Several major differences in the design and outcomes of the two studies might explain their divergent conclusions.

In Orngreen's study, assessments of therapeutic effects were based on two exercise cycle tests during each treatment period (placebo or bezafibrate), using what was presented as two "primary" outcomes, namely whole body FAO measured by indirect calorimetry, and heart rate $(\mathrm{HR})$, at exercise. There was no improvement in FAO in patients receiving bezafibrate, compared to placebo, in these experiments. This is in apparent contrast with the results of the first trial, in which biochemical analysis revealed a substantial increase in skeletal muscle FAO capacities in CPT2-deficient patients receiving bezafibrate. According to Orgreen et al., the absence of changes in FAO would be due to a negative influence of bezafibrate on lipolysis, the main determinant of whole body FAO, as reflected by the low plasma free fatty acid levels in the treated patients.

However, an alternative explanation exists. Indeed, it can be noticed that exercise tests were performed in patients exhibiting high levels of plasma insulin, which is known to act as a powerful inhibitor of lipolysis. This is particularly apparent during the bezafibrate period, in which insulinemia reached levels ( $>90$ picomol/l) that could induce a $>75 \%$ inhibition of lipolysis (10). There is, to our knowledge, no literature data indicating that high insulin levels could be ascribed to the drug itself, which was reported either to have no effect or to improve 
insulin sensitivity $(11,12)$. However, to directly test this assumption, we measured plasma insulin in the six CPT2-deficient patients included in our initial trial. Insulin levels were determined in frozen plasmas sampled after overnight fasting, prior to- and at the end of the bezafibrate treatment (table 1). As can be seen, these patients exhibited insulinemia 1.5-fold to 3-fold lower than patients in the Orngreen study, and these were found unchanged under bezafibrate. Accordingly, high insulinemia in the Danish study could not be due to the drug treatment but appear consistent with the fed state of patients at the time of exercise (2-3 hours after a breakfast of unknown composition). These high insulin levels could have exerted strong negative effects on lipolysis, hence hampering any increase of FAO, and masking the effects of bezafibrate.

The second "primary" outcome chosen in this trial was HR, based on the author's statement that bezafibrate "should lower HR during exercise, which would indicate improved exercise tolerance". There is, however, no rationale for this assumption. At first, the reference provided to support this statement deals with patients with Mac Ardle disease, hence is not relevant for CPT2 or VLCAD-deficient patients (13). Second, treatment by fibrate has never been reported to lower HR at exercise. Second, and more importantly, this choice is in contradiction with previous studies from Orngreen et al showing that HR profiles during cycling tests were fully normal in CPT2- and in VLCAD-deficient patients $(14,15)$. Thus, HR cannot be expected to change on treatment, and, accordingly, is not a clinically meaningful endpoint to evaluate bezafibrate effects in these patients.

Incidentally, a similar critique applies to the choice of perceived rate of exertion (Borg rate) as a secondary endpoint. Indeed, in the same previous studies, Borg rates during cycling tests were also reported to be normal in CPT2- or VLCAD-deficient patients. Accordingly, neither HR nor Borg rate values could provide any information on possible effects of the drug on exercise tolerance, contrary to the assumptions made by the authors.

Finally, one of the main questions is to know whether, as suggested by the first trial, bezafibrate treatment could improve the muscular manifestations of the disorders, including myalgia, muscle weakness, muscle stiffness, and rhabdomyolysis episodes, which significantly impact patients' quality of life and physical activity. Surprisingly, these symptoms and their possible changes in response to treatment were barely addressed in the Orgreen report, which reported very limited clinical data. For example, there was no mention of patients' muscular pain, muscular handicap, or physical limitation, in daily life or at exercise, during the trial period. To the exception of one value, Creatine kinase (CK) plasma levels were clearly in the low range for this kind of disorders in all the patients. Nine out of ten patients did not suffer any episode of myoglobinuria (only one attack reported in one patient). It was thus impossible to evaluate a possible reduction in the frequency of myoglobinuria episodes, yet this was presented as a secondary endpoint.

By comparison, the six CPT2-deficient patients considered in our initial trial regularly complained of myalgia, and exhibited rhabdomyolysis episodes before and during the trial period, generally triggered by moderate physical activity $(7,8)$. This led us to rule out exercise tests in the design of our trial. Importantly, treatment by bezafibrate led to a clear decline in myalgia, and reduced the frequency, severity and duration of rhabdomyolysis episodes in all the CPT2-deficient patients, in this initial trial. This went together with improvements in physical activity scores in the SF 36 survey, whereas the Bouchard 
questionnaire revealed no change under bezafibrate in the second trial. Clinical manifestations of CPT2- or VLCAD-deficiency are known to exhibit a large inter-individual variability, but it appears that the patients considered in the Orngreen trial likely exhibited very mild disease symptoms, with few, if any, signs of exercise intolerance or physical limitations. It is unfortunate to focus on such mild phenotypes since this makes it very difficult to demonstrate any clinical benefit of bezafibrate treatment, and (even more importantly) to extrapolate to patients with more severe phenotypes.

Overall, these limitations clearly call into question the conclusions reached by Orngreen that bezafibrate is ineffective in adults with CPT2 and VLCAD deficiencies. On the contrary, it is conceivable that possible beneficial effects of bezafibrate on muscular FAO and diseases symptoms could not be revealed in this study, due to inadequate study design and evaluation criteria, and to the phenotypes of study participants.

It can also be regretted that no data was provided on patients cell response to bezafibrate, as well as on patient genotypes, since this precludes any conclusion on the translation of exvivo studies into clinics. As a matter of fact, evaluation of new therapies in these heterogeneous diseases will obviously gain to integrate molecular and cellular data that might provide companion diagnostic tests, in parallel with accurate analysis of disease natural history, in order to precisely assess the eventual clinical benefit. In this regards, the relatively limited number of patients in these disorders should represent an incentive for personalized medicine, in order to improve treatment effectiveness.

Finally, the questions of trial design, and of the choice of clinically meaningful outcomes, are particularly complex for FAO disorders, as well as for many other diseases of mitochondrial energy metabolism. Despite these hurdles, it is absolutely essential to encourage clinical research in these areas. As for many other drugs, definite conclusions on the potential of bezafibrate in FAO disorders will require performing a number of trials, and, eventually, to reconcile apparently discordant data. Apparently negative studies can also suggest the need for further research with more refined methods (16).

We hope that the present comments will contribute to maintain our efforts in this direction, in order to progress in the treatment of these painful and incapacitating diseases with no pharmacological therapy to date. 


\section{References}

1 Froyland, L., Madsen, L., Vaagenes, H., Totland, G.K., Auwerx, J., Kryvi, H., Staels, B. and Berge, R.K. (1997) Mitochondrion is the principal target for nutritional and pharmacological control of triglyceride metabolism. J Lipid Res, 38, 1851-1858.

2 Minnich, A., Tian, N., Byan, L. and Bilder, G. (2001) A potent PPARalpha agonist stimulates mitochondrial fatty acid beta-oxidation in liver and skeletal muscle. Am J Physiol Endocrinol Metab, 280, E270-279.

3 Djouadi, F. and Bastin, J. (2008) PPARs as therapeutic targets for correction of inborn mitochondrial fatty acid oxidation disorders. J Inherit Metab Dis.

$4 \quad$ Li, H., Fukuda, S., Hasegawa, Y., Kobayashi, H., Purevsuren, J., Mushimoto, Y. and Yamaguchi, S. (2010) Effect of heat stress and bezafibrate on mitochondrial beta-oxidation: comparison between cultured cells from normal and mitochondrial fatty acid oxidation disorder children using in vitro probe acylcarnitine profiling assay. Brain Dev, 32, 362-370.

5 Yamaguchi, S., Li, H., Purevsuren, J., Yamada, K., Furui, M., Takahashi, T., Mushimoto, Y., Kobayashi, H., Hasegawa, Y., Taketani, T. et al. (2012) Bezafibrate can be a new treatment option for mitochondrial fatty acid oxidation disorders: evaluation by in vitro probe acylcarnitine assay. Mol Genet Metab, 107, 87-91.

6 Yasuno, T., Osafune, K., Sakurai, H., Asaka, I., Tanaka, A., Yamaguchi, S., Yamada, K., Hitomi, H., Arai, S., Kurose, Y. et al. (2014) Functional analysis of iPSC-derived myocytes from a patient with carnitine palmitoyltransferase II deficiency. Biochem Biophys Res Commun, 448, 175-181.

7 Bonnefont, J.P., Bastin, J., Behin, A. and Djouadi, F. (2009) Bezafibrate for treatment of an inborn mitochondrial ß-oxidation defect. N Engl J Med, 360, 838-840.

8 Bonnefont, J.P., Bastin, J., Laforet, P., Aubey, F., Mogenet, A., Romano, S., Ricquier, D., Gobin-Limballe, S., Vassault, A., Behin, A. et al. (2010) Long-term follow-up of bezafibrate treatment in patients with the myopathic form of carnitine palmitoyltransferase 2 deficiency. Clin Pharmacol Ther, 88, 101-108.

9 Orngreen, M.C., Madsen, K.L., Preisler, N., Andersen, G., Vissing, J. and Laforet, P. (2014) Bezafibrate in skeletal muscle fatty acid oxidation disorders: a randomized clinical trial. Neurology, 82, 607-613.

10 Stumvoll, M., Jacob, S., Wahl, H.G., Hauer, B., Loblein, K., Grauer, P., Becker, R., Nielsen, M., Renn, W. and Haring, H. (2000) Suppression of systemic, intramuscular, and subcutaneous adipose tissue lipolysis by insulin in humans. J Clin Endocrinol Metab, 85, 3740-3745.

11 Tenenbaum, A. and Fisman, E.Z. (2012) Fibrates are an essential part of modern anti-dyslipidemic arsenal: spotlight on atherogenic dyslipidemia and residual risk reduction. Cardiovasc Diabetol, 11, 125.

12 Tremblay-Mercier, J., Tessier, D., Plourde, M., Fortier, M., Lorrain, D. and Cunnane, S.C. (2010) Bezafibrate mildly stimulates ketogenesis and fatty acid metabolism in hypertriglyceridemic subjects. J Pharmacol Exp Ther, 334, 341-346.

13 Vissing, J. and Haller, R.G. (2003) The effect of oral sucrose on exercise tolerance in patients with McArdle's disease. N Engl J Med, 349, 2503-2509.

14 Orngreen, M.C., Duno, M., Ejstrup, R., Christensen, E., Schwartz, M., Sacchetti, M. and Vissing, J. (2005) Fuel utilization in subjects with carnitine palmitoyltransferase 2 gene mutations. Ann Neurol, 57, 60-66. 
15 Orngreen, M.C., Norgaard, M.G., Sacchetti, M., van Engelen, B.G. and Vissing, J. (2004) Fuel utilization in patients with very long-chain acyl-coa dehydrogenase deficiency. Ann Neurol, 56, 279-283.

$16 \square$ Knopman D.K., Gross R.A. (2014) A call for "negative" outcome studies.

Neurology, 82,11-12.

Table 1. Plasma insulin in patients before and after 6-month-treatment with bezafibrate

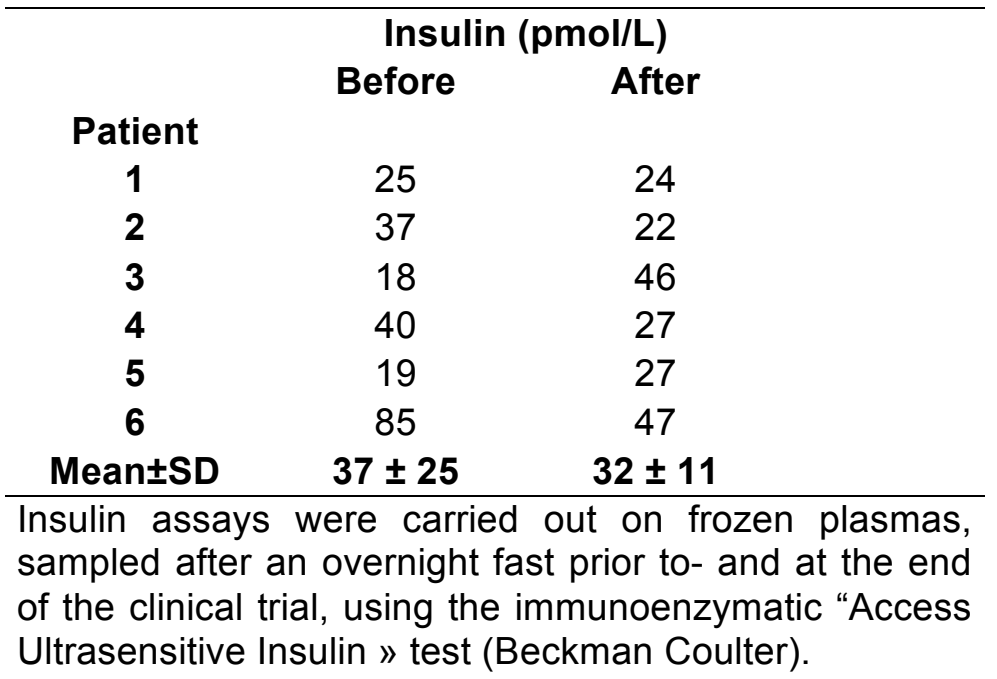

